\title{
EL PROCESO DE POBLAMIENTO EN MISIONES-ARGENTINA: UN ABORDAJE A PARTIR DE LA OBRA DE ROBERT EIDT
}

\author{
The settlement process in Misiones-Argentina: \\ an approach based on the work of Robert Eidt
}

\author{
Zang, Laura Mabel* \\ https://orcid.org/0000-0002-5384-6654
}

\section{Resumen}

El presente trabajo constituye un análisis crítico del libro Pioneer Settlement in Northeast Argentina, escrito por el geógrafo alemán Robert C. Eidt en 1971. Reconociendo este material como clave para el estudio de la problemática del poblamiento de Misiones, el autor analiza este proceso a lo largo de varios siglos de historia abordando los factores intervinientes en dicho fenómeno, las relaciones de poder implícitas, los cambios espaciales suscitados y los actores involucrados. Al mismo tiempo, el libro presenta puntos de contacto entre varias disciplinas: historia, geografía, economía, política y biología y representa una referencia prácticamente obligada para toda investigación que centre su interés ya sea en el poblamiento de Misiones como en la evolución del principal cultivo de esas tierras: la yerba mate.

$<$ Poblamiento $><$ Misiones $><$ Colonización $>$

\begin{abstract}
The present work constitutes a critical analysis of the book Pioneer Settlement in Northeast Argentina, written by the German geographer Robert C. Eidt in 1971. This book is central to the study of the settlement of Misiones. The author analyzes this process over several centuries of history, addressing the factors involved in this phenomenon, the implicit power relations, the spatial changes generated, and the actors involved. In addition, the book combines disciplines such a History, Geography, Economics, Politics, and Biology. It also represents a practically obligatory reference for any research that focuses its interest on either the settlement of Misiones or the evolution of the main cultivation of these lands: yerba mate.
\end{abstract}

$<$ Settlement $><$ Misiones $><$ Colonization $>$

Recibido: 20/10/2020 // Aceptado: 20/08/2021

\footnotetext{
* Becaria Posdoctoral (IESyH/CONICET). Doctora en Geografía por la Universidad Nacional del Nordeste y Magíster en Historia egresada de la Universidad Nacional de Tres de Febrero.
} 
A principios de la década de 1970, Robert C. Eidt ${ }^{1}$-geógrafo de origen alemán que se desempeñaba como Profesor en la Universidad de Wisconsin-Milwaukee [EE. UU] - escribió Pioneer Settlement in Northeast Argentina [Asentamientos pioneros en el Noreste de Argentina]. El estudio presentado como libro tuvo, sin embargo, un primer antecedente con la publicación en 1965 en Alemania del artículo "Die Staatlishe und Private Besiedlung von Misiones (Argentinien)" [El Estado y los asentamientos privados en Misiones (Argentina)], donde analizaba la consolidación de las colonias surgidas a partir de la intervención de capitales privados en el Alto Paraná.

El libro de Eidt forma parte de una colección mayor de estudios editados por la Universidad de Wisconsin que centraron su atención en regiones inhóspitas o de escaso poblamiento a lo largo de América, Asia y África. Dentro de este marco, en 1962, Perry McDonough Collins publicó Siberian Journey: Down the Amur to the Pacific 18561857; en 1966, Marvin P. Miracle escribió Maize in tropical África; en 1968, Desert and River in Nubia; Geomorphology and Prehistoric Environments fue publicado por Karl W. Butzer; en 1969, Frederik J. Simoons publicó Northwest Ethiopi, peoples and economy; también en el mismo año, Robert Eric Frykenberg presentó Land Control and Social Structure in Indian History.

Imagen 1. Libro de Robert Eidt

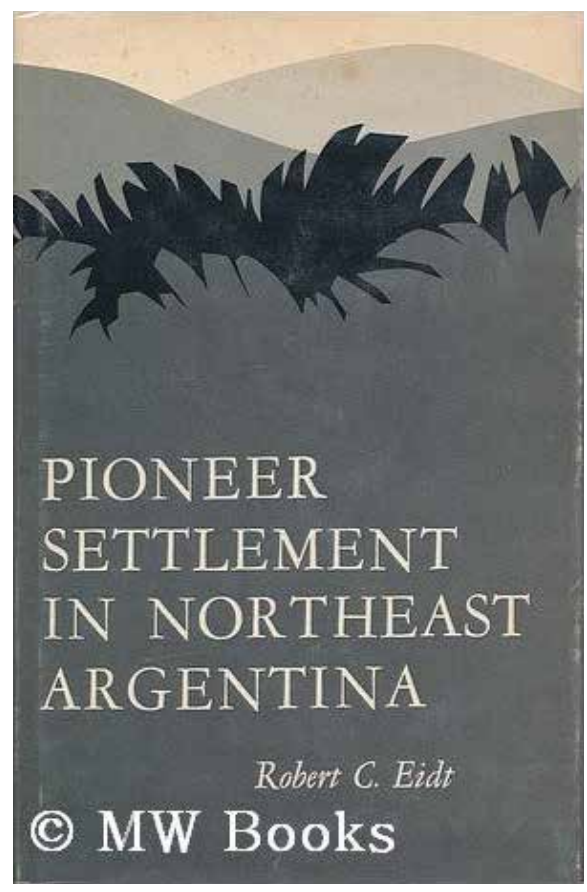

${ }^{1}$ El mismo autor también publicó Pionner Settlement in Eastern Perú en 1962; Man, Culture and Settlement. A Festschrift to R. L. Singh en 1977 y Advances in Abandoned Settlement Analysis: Application to Prehistoric Anthrosols in Colombia, South America en 1984. 
Pioneer Settlement in Northeast Argentina puede ser considerado precursor en el estudio del poblamiento de Misiones, el arribo de inmigrantes y la consolidación de colonias en el territorio como también las particularidades de los patrones de asentamiento. Para comienzos de la década de 1970 en que el libro de Eidt fue publicado, la problemática del poblamiento y la afluencia de inmigrantes hacia Misiones no estaban aún estudiados académicamente. Recién tras la creación de la Universidad Nacional de Misiones (UNaM) en el año 1973, se conformaron espacios de análisis y discusión del proceso colonizador desde una perspectiva antropológica e histórica ${ }^{2}$.

Producto de un profundo trabajo de campo efectuado en Misiones entre 1964 y $1967^{3}$, Robert Eidt remarca la posición fronteriza de Misiones y la condición de espacio disponible para la consecución de un plan de colonización territorial. En ese sentido, el estudio del autor centra su atención en el proceso de poblamiento de Misiones producido a lo largo de varios siglos de historia analizando los factores intervinientes en dicho fenómeno, las relaciones de poder implícitas, los cambios espaciales suscitados y los actores involucrados. Las fuentes utilizadas se encuentran en estrecha relación con los períodos históricos abordados y entre ellos podemos citar a las crónicas escritas por distintos viajeros, agrimensores y estadistas vinculados a Misiones.

Para una mejor comprensión de los procesos analizados en el libro, el presente trabajo fue estructurado en cinco ejes temáticos que marcan, en sentido cronológico, las fases del poblamiento de Misiones. La primera parte es más general y aborda las condiciones naturales del territorio y las posibilidades para el establecimiento de inmigrantes. El segundo apartado, analiza los primeros antecedentes de la presencia de población estable en la región suscitado a partir de la consolidación de las Misiones Jesuíticas. En el tercer apartado, el foco de atención fue puesto en la alta conflictividad en la que recayó el espacio tras la expulsión de los Padres Jesuitas, las pujas por el control del territorio misionero para finalmente abordar el tema de la creación del Territorio Nacional de Misiones. El apartado cuatro, estudia la conformación de las colonias en tierras del fisco y el establecimiento de inmigrantes europeos a partir del

2 Dentro de este marco, podemos citar la tesis doctoral en Antropología Sociocultural efectuada por Leopoldo Bartolomé y que fue defendida en la Universidad de Wisconsin (EE.UU) en 1974 donde analizó las migraciones galitzianas (polacas y ucranianas), sus estrategias de adaptación y el rol de la etnicidad de los eslavos en la formación de la colonia Apóstoles - situada en el sur de Misiones-; este estudio, sin embargo, fue publicado por primera vez como libro recién en el año 2000. En otro trabajo, Leopoldo Bartolomé (1982) realizó un estudio de carácter más general centrando su atención en el desarrollo agrícola de Misiones; por su parte, Perié de Schiavoni y Souvi (1985) analizaron el fenómeno de las migraciones hacia Misiones desde una perspectiva general. Presenta una centralidad destacada para el abordaje del problema de la yerba mate y su influencia en el proceso del poblamiento del entonces Territorio Nacional de Misiones, la tesis doctoral de Alfredo Bolsi (1986) "Misiones (una aproximación geográfica al problema de la yerba mate y sus efectos en la ocupación del espacio y el poblamiento". Todos estos estudios emplearon como material de consulta el trabajo de Robert Eidt.

3 Durante su estadía en Misiones, Robert Eidt estuvo en contacto con Miguel Ángel Stefañuk -reconocido geógrafo misionero-con quien intercambió distintos aspectos de su investigación (Entrevista a Élida Arehardt). 
Zang. El proceso de poblamiento en Misiones-Argentina: un abordaje a partir de la obra de Robert Eidt.

impulso estatal. Finalmente, el quinto apartado aborda la conformación de las colonias privadas del Alto Paraná y las particularidades de su organización.

\section{Acerca de las características topográficas y climáticas de Misiones}

En la primer parte del libro, el geógrafo ubica a Misiones dentro de un territorio más amplio -el argentino e incluso americano- y señala que este escenario constituye una zona de investigación única por las características fisiográficas que presenta y por la particular evolución en el proceso de su poblamiento. Al igual que en otros lugares del país como la Patagonia, Chaco y La Pampa, el sector dirigente nacional desalentó en atraer a esas tierras "a sus propios ciudadanos [y] ofreció tierras de Misiones a bajos precios a los europeos que querían superar las tensiones de la Primera Guerra Mundial" (Eidt, 1971: 9).

El autor sitúa geográficamente a Misiones y realiza una detallada exposición acerca de las particularidades climáticas de la provincia, características de los suelos, recursos naturales disponibles y topografía con el objetivo de demostrar que el territorio de Misiones presenta aptitud natural para sustentar un importante núcleo poblacional. En este sentido, a diferencia de otros territorios del país abiertos a la colonización, identificó una serie de problemas que necesariamente deben ser tenidos en cuenta para el desarrollo del proceso colonizador: el clima cálido y la exuberante vegetación propician la presencia de serpientes venenosas y de "insectos picadores"; al mismo tiempo, las "temperaturas extremas" constituyen un gran inconveniente para los "colonos que no han sido capacitados en métodos agrícolas semitropicales" (Eidt, 1971:15). Si bien el invierno no es intenso, Eidt señala que entre mediados de mayo y mediados de agosto las heladas también pueden ocasionar inconvenientes, principalmente en las zonas elevadas de la provincia. Por su parte, las intensas precipitaciones pueden ocasionar el desborde de los ríos y detener la circulación por estos medios; sin embargo, señala el autor que en el Alto Paraná el aislamiento fue solucionado de manera gradual con el avance en la construcción de puentes y carreteras.

Pese a estos factores adversos, a partir del análisis detallado de la documentación acerca de las condiciones naturales imperantes en Misiones ${ }^{4}$, en el relato de Eidt sobresalen las múltiples ventajas que tiene el territorio y que representan un contraste con la visión del "infierno verde" que percibían muchos de los europeos (Eidt, 1971: 13). Dividido en distintas regiones naturales, Misiones cuenta con un clima apto para el desarrollo de actividades agrícolas y ganaderas con numerosos afluentes de agua para consumo humano y animal, suelos sumamente fértiles y una importante variedad de recursos naturales.

\footnotetext{
4 Véase Misiones Provincia, Planeamiento de la Provincia de Misiones (Buenos Aires, 1961); Misiones Provincia, Informe Edafológico de Misiones (Buenos Aires, 1964); Misiones Provincia, Informe sobre los recursos forestales (Buenos Aires, 1964) y Misiones Provincia, Informe Geológico (Buenos Aires, 1964).
} 


\section{El poblamiento de Misiones en el periodo de las Misiones Jesuíticas: un primer antecedente de la presencia en la región}

La temprana presencia de europeos en la región es analizada por Eidt en el capítulo titulado "Colonial Land Opening in Misiones: The Beginning of European Settlement", centrando especialmente su atención en la consolidación de las Misiones Jesuíticas en Misiones. El establecimiento de los jesuitas en ese territorio se remonta a finales del siglo XVII cuando dieron inicio a su misión evangelizadora en una región más amplia conformada por un grupo de treinta pueblos situados en las zonas fronterizas de los países de Paraguay, Brasil, Argentina y Uruguay. Organizados económicamente con una estructura centralizada, siguieron ciertas pautas ya practicados por los pueblos guaraníes donde se podía identificar una modalidad de producción familiar y otra comunal con bienes obtenidos a partir de allí para la subsistencia del núcleo familiar. Entre los cultivos el autor menciona citrus, tabaco, maíz, batatas, melones, caña de azúcar, algodón y mandioca; estos, junto a la ganadería, ayudaban al sostenimiento del núcleo poblacional.

Interesa aquí también no solo qué cultivaban sino cómo lo hacían: la limpieza de tierras donde volteaban la vegetación con hachas para luego quemar los restos y labrar la tierra es un método "de siembra y arado [que] aún se siguen en Misiones" (Eidt, 1971: 46). Por su parte, el establecimiento de las misiones jesuíticas representó una fuerte influencia en las formas de asentamiento moderno que las precedieron.

En una posición distinta a la del resto de los cultivos, el autor ubica a la yerba mate pues este era un recurso con el cual obtenían dinero a partir de su comercialización:

Gradualmente, esto se convirtió en un negocio lucrativo porque los jesuitas pudieron vender el producto excedente en Asunción, donde tenía mucha demanda, y más tarde en Santa Fe y Buenos Aires. Uno de los mercados más grandes se desarrolló en el Alto Perú, donde los indios trabajadores de las minas de Potosí solicitaban la bebida [...]" (Eidt, 1971; 44).

El logro de la reproducción de la yerba mate por los jesuitas representó un antes y un después en la organización económica y en la forma del trabajo destinado a su explotación. En efecto, Eidt (1971: 44) señala que "la creciente importancia económica de la yerba mate y el riesgo creciente de perder a los indígenas que recolectaban las hojas silvestres, llevó a los jesuitas a limpiar la tierra y criar yerba mate de manera eficiente en un sistema que desapareció cuando los jesuitas se fueron". El monopolio del cultivo yerbatero se convirtió en el más importante pilar económico de las reducciones ${ }^{5}$; sin embargo, junto a otros factores ${ }^{6}$ la gran prosperidad alcanzada por los jesuitas

\footnotetext{
5 Según estimaciones del autor, por misión las cosechas eran de alrededor de 1300 arrobas.

${ }^{6}$ Entre ellos, el autor menciona quejas contra los jesuitas por no enseñar a los guaraníes la "lengua de Cervantes" y por la presencia de "demasiados sacerdotes extranjeros que administraban a los nativos" (Eidt, 1971: 49). Al mismo tiempo, entre 1764 y 1766, grandes epidemias de viruela diezmaron a la población indígena.
} 
Zang. El proceso de poblamiento en Misiones-Argentina: un abordaje a partir de la obra de Robert Eidt.

generó suspicacias entre distintos sectores allegados a la corona y entre otros grupos congregacionales terminando en la definitiva decadencia del sistema jesuítico.

\section{Las disputas por Misiones: desde la expulsión de los padres jesuitas a la creación del Territorio Nacional de Misiones}

En el estudio de la literatura existente y a partir del análisis de fuentes documentales respecto al período jesuítico, su decadencia y el largo camino recorrido hasta la creación del Territorio Nacional de Misiones en el año 1881, el autor presta especial atención a las relaciones de poder y las disputas suscitadas para el control de este escenario. De este modo, el período comprendido entre la segunda mitad del siglo XVIII -en 1767 se produjo la expulsión de los padres jesuitas de las reduccioneshasta finales del siglo XIX con la creación del Territorio Nacional de Misiones fue de gran conflictividad: las constantes incursiones paraguayas sobre el departamento misionero de Candelaria y la Guerra contra el Imperio de Brasil entre 1825 y 1826, provocaron un paulatino despoblamiento de Misiones y la dispersión de sus habitantes. Al mismo tiempo, a esta difícil situación política y militar, deben sumársele los cambios en su dependencia administrativa pasando a estar entre 1815 a 1821 bajo jurisdicción artiguista, seguida de un breve período de integración a la provincia de Entre Ríos liderado por Ramírez e inmediatamente pasó a estar bajo protección santafesina (1822).

Después de 1832, la antigua provincia de Misiones fue disuelta pasando a integrar a la provincia de Corrientes. El Estado nacional no demostró gran interés en la administración de estas tierras hasta después de la "Guerra de la Triple Alianza" o "Guerra del Paraguay" (1865-1870) -conflicto que enfrentó a Argentina, Brasil y Uruguay contra Paraguay-, momento en que los dirigentes nacionales comenzaron a manifestar su preocupación acerca de la importancia de Misiones, la fuente de recursos naturales que contenía y su particular posición geográfica fronteriza; en este sentido, el autor evidencia que la creación del Territorio Nacional de Misiones en el año 1881 fue una respuesta a toda esta situación. El Estado nacional intentó, tras este importante hecho, llevar adelante un plan colonizador a partir del arribo de colonos europeos.

Tras conocer la existencia del proyecto de ley de creación del Territorio Nacional, el gobierno de Corrientes se opuso fervientemente al mismo pues, de aprobarse, la provincia perdería el usufructo de los recursos misioneros del que disponían desde 1832. En junio de 1881, ante el panorama de una inevitable Federalización de Misiones (Zang, 2020), los dirigentes de Corrientes autorizaron una rápida venta de tierras consolidándose, según Robert Eidt, una "estructura agraria latifundista" que frenó el efectivo progreso del proceso colonizador.

Para analizar este complejo proceso, Robert Eidt se valió de un importante apartado cartográfico que sitúa a Misiones dentro de un escenario de gran dinamismo con cambios limítrofes significativos y con transformaciones en su estructura económica y política. De este modo, mientras en el mapa 1 el Territorio Nacional aún presenta las tierras que eran objeto de disputa con Brasil, en el mapa 2 vemos una redefinición de los límites territoriales tras el laudo de Cleveland en 1895. 
Mapa 1. Venta de tierras efectuada por la provincia de Corrientes ante la Federalización

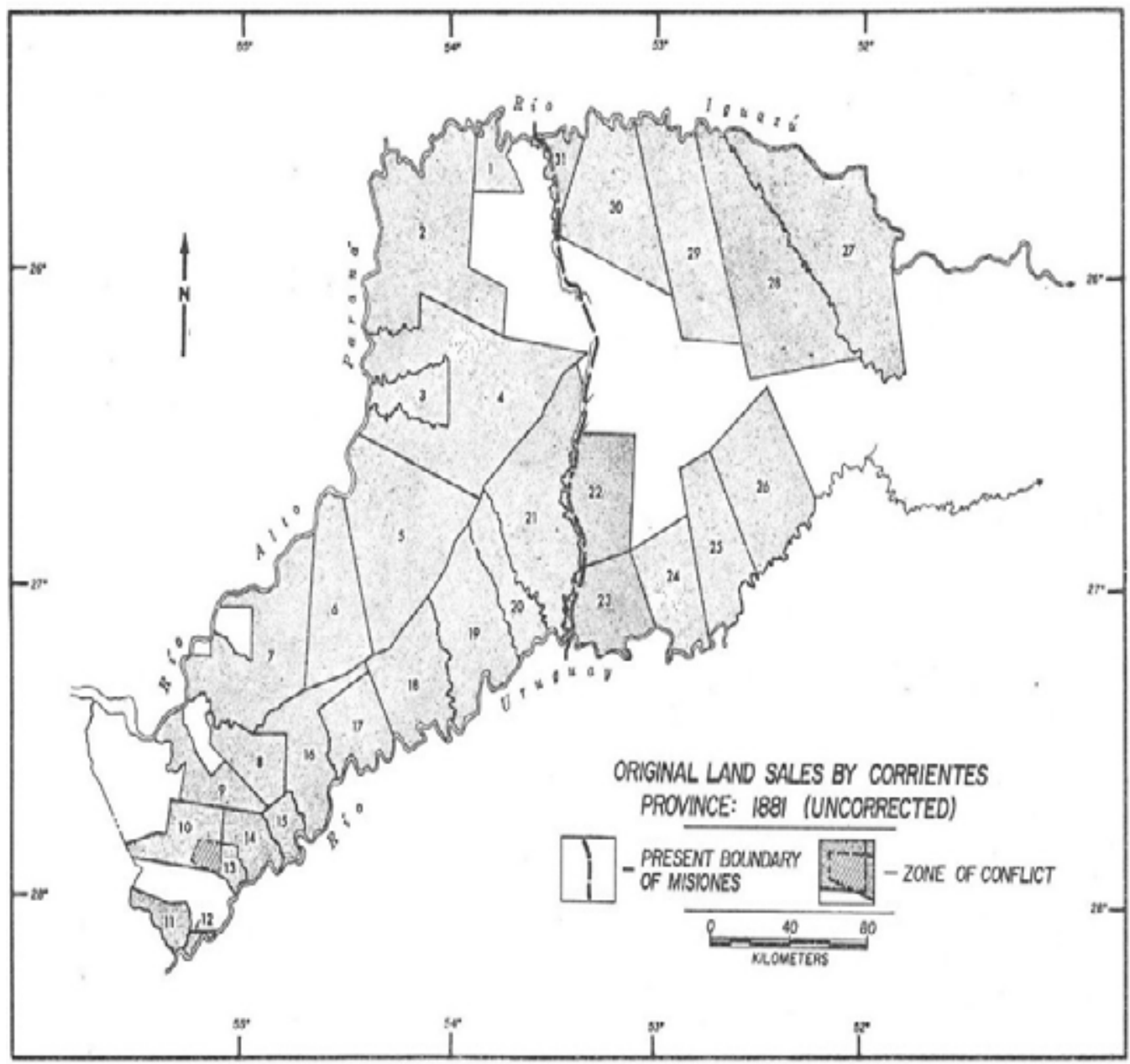

Fuente: Eidt, 1971: 74.

Para finales del siglo XIX, el autor sitúa a los principales núcleos de población en tierras fiscales ${ }^{7}$ : Concepción, San Javier y Monteagudo en el Alto Uruguay; al sur: Apóstoles y San Carlos, y en el Alto Paraná: Candelaria, Santa Ana, San Ignacio, Loreto y Corpus. En la siguiente tabla, Eidt expone la composición de la población de las colonias de acuerdo al origen nacional y la cantidad de hectáreas cultivadas.

7 Estas eran las tierras que escaparon a la "venta" efectuada por Corrientes y quedaron debido a esa situación, bajo dominio del gobierno del recientemente creado Territorio Nacional de Misiones. 
Zang. El proceso de poblamiento en Misiones-Argentina: un abordaje a partir de la obra de Robert Eidt.

Mapa 2. Venta de tierras con correcciones tras el laudo Cleeveland

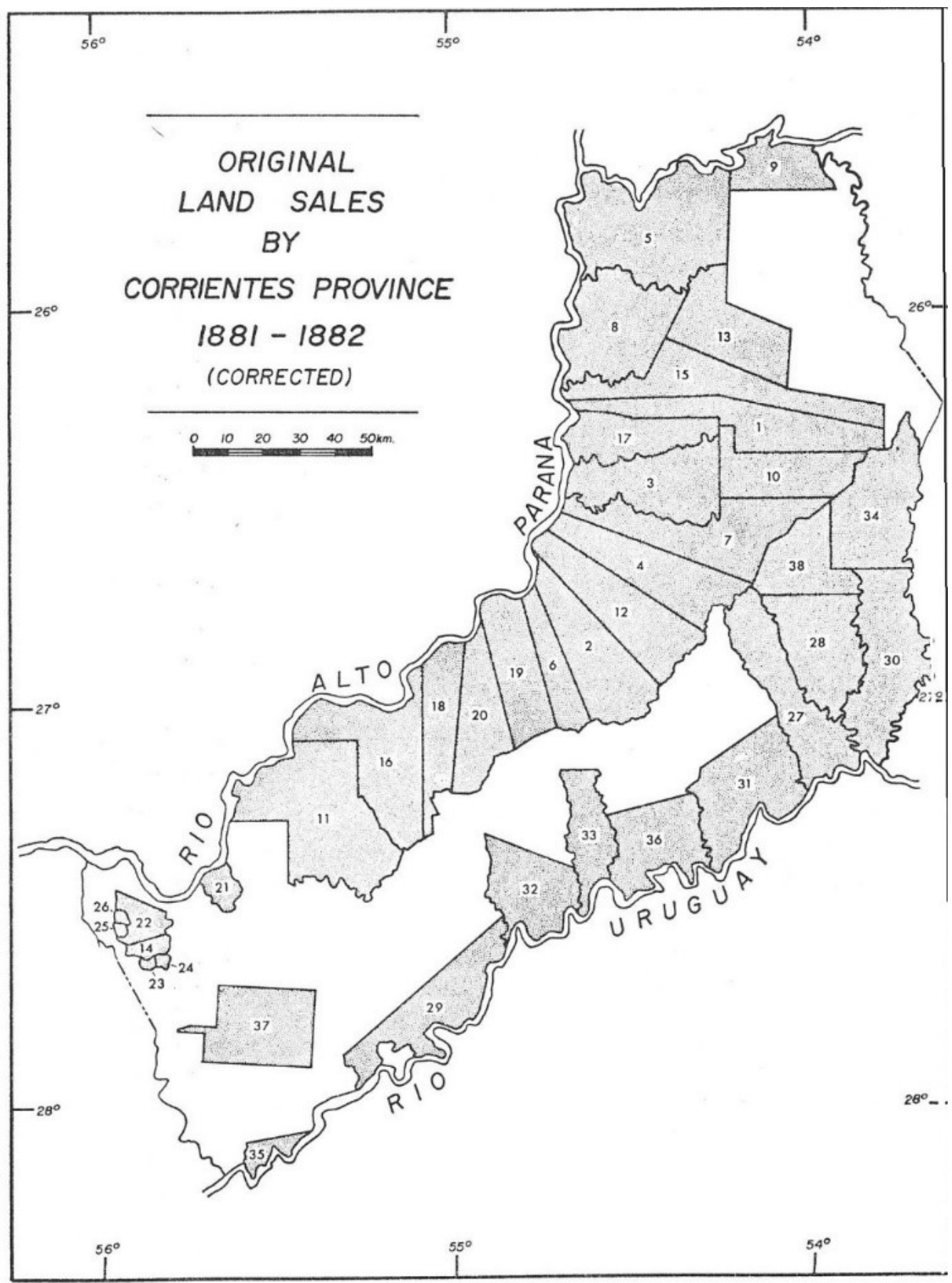

Fuente: Eidt, 1971: 76. 
Tabla 1. Colonización extranjera en Misiones, 1903

\begin{tabular}{cccc}
\hline País de origen & Familias & Ha. Cultivadas & $\begin{array}{c}\text { Promedio Ha por } \\
\text { familia }\end{array}$ \\
\hline Polonia & 810 & 6171 & 7,6 \\
\hline Brasil & 502 & 2544 & 5,1 \\
\hline Paraguay & 116 & 458 & 3,9 \\
\hline Italia & 72 & 584 & 8,1 \\
\hline Rusia & 70 & 363 & 5,2 \\
\hline Alemania & 59 & 322 & 5,4 \\
\hline Espana & 38 & 240 & 6,3 \\
\hline Francia & 30 & 195 & 6,5 \\
\hline Suecia & 15 & 46 & 3,1 \\
\hline Oriente & 10 & 54 & 5,4 \\
\hline Suiza & 6 & 55 & 9,8 \\
\hline Arabia & 2 & 6 & 3,2 \\
\hline Dinamarca & 1 & 3 & 3,0 \\
\hline Inglaterra & 1 & 2 & 2,0 \\
\hline Grecia & 1 & 3 & 3,0 \\
\hline Norte América & 1 & 5 & 5,0 \\
\hline & & &
\end{tabular}

Fuente: Eidt, 1971: 93.

\section{La colonización en tierras fiscales}

Los emprendimientos colonizadores en tierras pertenecientes al fisco, tuvieron un nuevo impulso a partir de la intervención del Estado nacional tras la creación del Territorio Nacional en 1881. Como consecuencia de esto, el 20 de febrero de 1883, el gobierno decidió nacionalizar los asentamientos de Candelaria y Sana Ana a partir de su mensura; dicha tarea fue encargada a Rafael Hernández quien organizó un fraccionamiento de lotes en promedio de 25 hectáreas en forma de damero.

Para el año 1894 el gobierno nacional dejó sin efecto una parte de las "ventas" de tierras efectuadas en Misiones luego de la creación del Territorio Nacional en 1881 por no haber cumplido sus propietarios con la realización de las correspondientes mensuras. Estas tierras se convirtieron luego en los focos de asentamiento de los primeros grupos de inmigrantes europeos que llegaron al territorio misionero. Si bien estos antecedentes fueron importantes, la colonización oficial cobró más fuerza con la fundación de Apóstoles en el año 1897, que recibió a inmigrantes polaco-galitzianos a partir de las promociones realizadas por el entonces Gobernador del Territorio Nacional, Juan José Lanusse.

De este modo, para finales del siglo XIX, los principales núcleos poblacionales se encontraban en Concepción, San Javier y Monteagudo en el Alto Uruguay; Apóstoles y San Carlos en el sur y Candelaria, Santa Ana, San Ignacio y Corpus en el Alto Paraná. Estos asentamientos surgieron principalmente sobre la base de los antiguos pueblos jesuitas que escaparon a la "venta" de tierras realizada por Corrientes en 1881. La infraestructura abandonada de las antiguas reducciones propició el desarrollo posterior de los primeros poblados y sus plantaciones de naranja y yerba mate fueron un recurso de suma importancia para los colonos recién arribados. De este modo, los conocimientos 
Zang. El proceso de poblamiento en Misiones-Argentina: un abordaje a partir de la obra de Robert Eidt.

acumulados sobre el sistema productivo existente en las antiguas reducciones constituyeron el modelo agrícola para los colonos (Eidt, 1971).

La recuperación de la técnica de reproducción de plantines de yerba mate por medio de la siembra fue uno de los factores que motivó el arribo de inmigrantes de diversos orígenes. Según Eidt, esta técnica habría sido redescubierta en la colonia Nueva Germania de Paraguay, por un inmigrante de origen alemán de apellido Neuman. Esta situación marcó un antes y un después en el proceso de poblamiento de Misiones: el agotamiento de la yerba mate de origen silvestre que venía siendo sobreexplotada desde el fin de la Guerra de la Triple Alianza, planteó la necesidad de experimentar el cultivo de yerba mate para lograr su reproducción. Para el año 1903, "un agrónomo en San Ignacio importó las semillas tratadas de Nueva Germania y comenzó una nueva era de prosperidad en Misiones justo cuando el producto silvestre se estaba agotando" (Eidt, 1971: 103). La yerba mate presentaba una serie de ventajas frente a otros productos: al tratarse de una planta nativa perenne y que ya se encontraba adaptada al suelo y al clima, era mejor en la prevención de la erosión que los cultivos anuales.

\section{El impulso colonizador a partir de la intervención de capitales privados: formación de las colonias del Alto Paraná misionero}

La intervención de capitales privados y la consolidación de Compañías de Colonización en el territorio de Misiones es analizado por el autor en el capítulo "Foreign Colonization Companies in Misiones". Gran parte de las tierras que habían sido adquiridas tras la federalización de Misiones por distintos grupos de la elite gubernamental, fueron vendidas tras el agotamiento los recursos más importantes y de más fácil acceso del suelo misionero a empresarios colonizadores que llevaron a cabo el fraccionamiento y venta de lotes a distintos grupos de inmigrantes. Dentro de las colonias surgidas de este modo, el autor centró su atención en los asentamientos de Puerto Rico, Montecarlo y Eldorado. Estas tres colonias del Alto Paraná constituyen los mayores núcleos de concentración de personas de origen alemán. Sin embargo, en ellas es posible advertir una distinción interna de acuerdo a los lugares de procedencia: alemanes procedentes directamente de Alemania en el caso de Eldorado y un número importante de ellos en Montecarlo y alemanes-brasileros en Puerto Rico.

El proceso colonizador de Puerto Rico (1919) y Montecarlo (1920) estuvo a cargo de la Compañía Colonizadora Alto Paraná Culmey y Cía. dirigida por Carlos Culmey. En estas colonias, la distribución de los migrantes siguió pautas religiosas muy marcadas:

Desde el principio, Culmey recibió solicitudes de agricultores católicos y protestantes en Brasil, pero debido a su origen cultural alemán en el que la formación de aldeas se limitaba a miembros de una religión y otra, [Culmey] decidió admitir solo colonos católicos en Puerto Rico. Sin embargo, el 20 de marzo de 1920, inició otra colonia de pioneros protestantes mientras continuaba administrando Puerto Rico. El nuevo 
asentamiento estaba ubicado a $45 \mathrm{~km}$ río arriba de Puerto Rico y eventualmente fue nombrado Monte Carlo (Eidt, 1971: 128).

Al norte de estas colonias fundadas por Carlos Culmey, Adolfo Schwelm adquirió las tierras que darán nacimiento a la colonia Eldorado en 1919. Este empresario de origen alemán, ya tenía importantes actividades económicas en otros lugares del país (Santiago del Estero, La Pampa y Chaco) y en Paraguay; vinculado a la casa Rothschield, contaba con un sólido respaldo económico y contactos en Europa donde radicó agencias para acaparar a posibles migrantes. En el contexto de la Europa empobrecida tras la Primera Guerra Mundial, estos medios publicitarios empleados por Schwelm no tardaron en acaparar la atención de personas deseosas de migrar hacia lugares en que podrían convertirse en propietarios de las tierras que cultivaran. Sin embargo, "el reclutamiento principal se llevó a cabo en Buenos Aires [...] allí sus agentes conversaron sobre la colonia con los recién llegados y mostraron mapas y fotografías de Eldorado, así como una película de $16 \mathrm{~mm}$ de la vida en el nuevo asentamiento" (Eidt, 1981: 131); como fuentes prioritarias para el análisis de las actividades de Adolfo Schwelm y de la Compañía Eldorado, Colonización y Explotación de Bosques, Eidt realizó un minucioso recorrido por las publicaciones y campañas publicitarias que la empresa colonizadora emitió a los fines de atraer inmigrantes desde Europa.

En estas colonias del Alto Paraná predominó el loteamiento Waldhufendorf ${ }^{8}$ siendo una excepción la colonia San Alberto en Puerto Rico (véase mapa 3 y 4). Este sistema de herencia europea, presentaba ventajas muy importantes en la distribución de los recursos (agua principalmente) y en propiciar la comunicación al interior de las colonias (Gallero, 2009). Debido a ello, los colonos "rechazaron de inmediato la forma damero utilizada en Misiones e introdujeron el concepto de Waldhufendorf" (Eidt, 1971: 122).

Tras la caracterización de las particularidades de los movimientos colonizadores tanto estatales como fiscales, Eidt presenta un panorama sobre los distintos asentamientos de Misiones y la evolución de las actividades a ellos vinculados. En relación a ello, el autor señala que "no hay duda de que la apertura de tierras en esta parte remota de América Latina ya pasó el punto de ser autopropulsada" y agrega que en un 35\% del total de tierras de Misiones se encuentran efectivamente ocupadas (Eidt, 1971: 182).

Durante el proceso colonizador, Eidt reconoce cuatro etapas que "se reflejan en el tipo de combinación de cultivos y en la cantidad de terreno despejado" (Eidt, 1971: 182). En la primera de las etapas corresponde a los primeros contactos del colono con las tierras adquiridas; en este momento, la limpieza de un espacio dentro del monte para el cultivo de los alimentos indispensables para la subsistencia y la construcción de un refugio rudimentario llamado "rancho" fueron esenciales. La segunda etapa, ya implicó una ampliación de la superficie plantada, la introducción de cultivos de renta como el tabaco y la sustitución del rancho por una casa realizada por maderas. En la tercera es posible la identificación de casas construidas en ladrillos; aquí el autor

\footnotetext{
8 Wald (bosque, monte), hufen (franja estrecha de tierra), dorf (poblado, villa). Este modelo de parcelamiento de las tierras empleado en las colonias alemanas del sur de Brasil tiene profundas raíces en la Europa rural (Gallero, 2009).
} 
Zang. El proceso de poblamiento en Misiones-Argentina: un abordaje a partir de la obra de Robert Eidt.

observa la gradual sustitución de las plantaciones de tabaco por yerba mate. En la última etapa, comenzaron las construcciones de casas para las nuevas generaciones residentes en las chacras. Al mismo tiempo, es posible registrar una mayor diversificación de la producción con la introducción de plantaciones de tung, te y cítricos, además de hortalizas; esta diversificación se produjo como una consecuencia de las "malas condiciones de mercado" de la yerba mate y a la crisis abatida sobre ese sector que culminó con la reglamentación del cultivo9 (Eidt, 1971: 183).

\section{Mapas 3 y 4. Sistema de parcelamiento Waldhufendorf predominante en las colonias del Alto} Paraná: Puerto Rico y Montecarlo
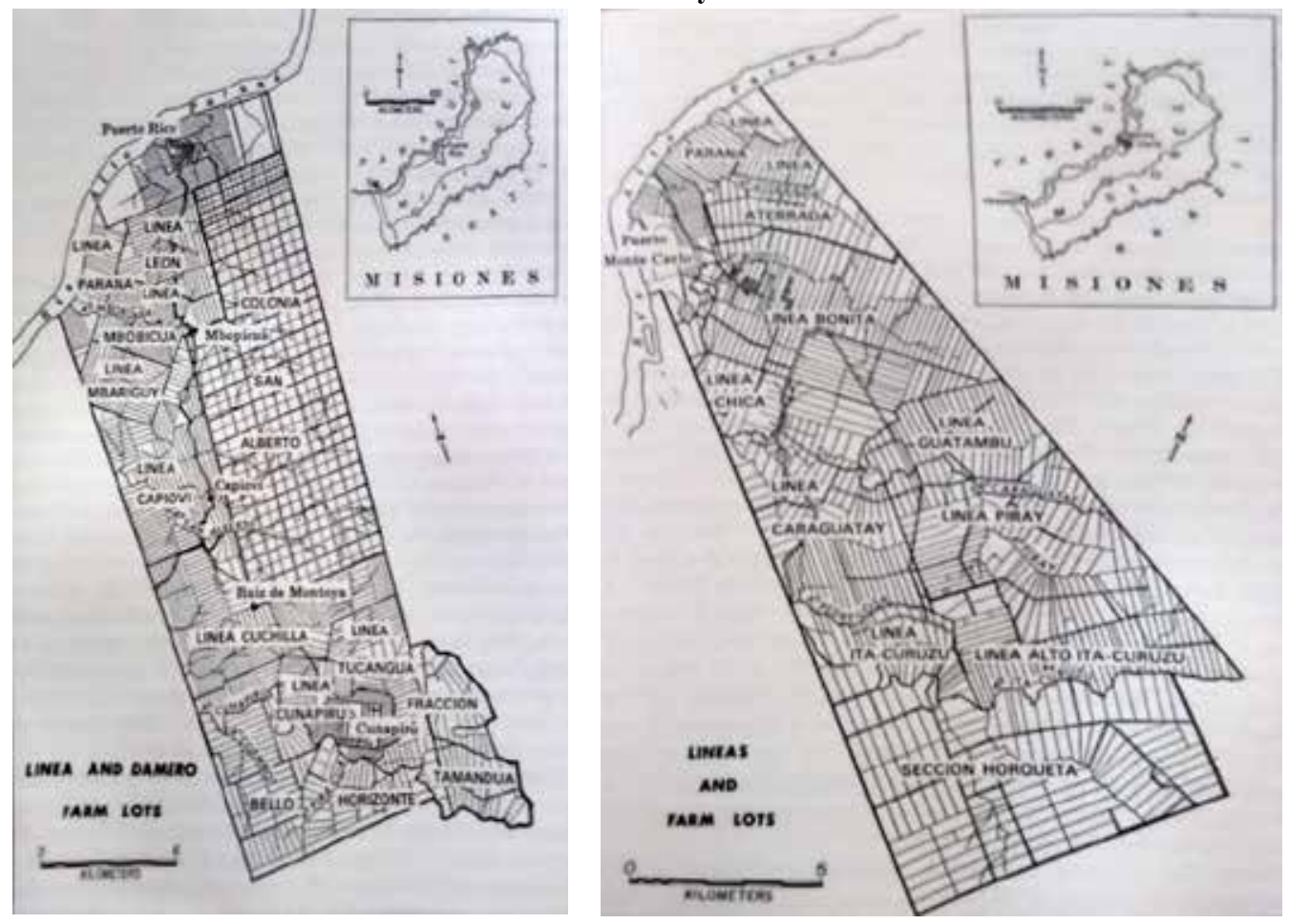

Fuente: Eidt, 1971:126-127

\section{A modo de conclusión}

Robert Eidt sostiene que su investigación sobre Misiones evidencia que se lograron "resultados" en el proceso de poblamiento y en el éxito de estos asentamientos. Esta situación dependió de una serie de factores como la ubicación de las áreas de colonización, elección y origen de los pobladores, método de titulación de tierras y herencia, sistema de utilización de recursos, entre otros. Por su parte, los modelos

\footnotetext{
9 En el marco de una fuerte crisis dentro del sector yerbatero, 04 de octubre de 1935, por ley $\mathrm{N}^{\mathrm{o}} 12236$, fue creada la Comisión Reguladora de la Yerba Mate [CRYM]. El organismo ejerció un estricto control en el control de la cantidad de la yerba mate producida limitando las nuevas plantaciones y regulando las podas (Zang, 2020).
} 
de asentamiento de los colonos tuvieron estrecha vinculación con los lugares en que estos se asentaron y con su origen étnico. En el Alto Paraná por ejemplo, donde las colonias fueron creadas y administradas mayormente por capitales privados y con predominancia de inmigrantes de origen alemán brasilero y alemán, fue empleado en forma predominante el sistema de organización de lotes Waldhufendorf; el damero en tanto, fue más común en las colonias fiscales del sur y centro de Misiones.

El autor reconoce a su vez otros lugares de América Latina [Paraguay, Perú, Bolivia y parte de Brasil] donde las condiciones de colonización fueron similares a las encontradas en Misiones y en este sentido considera que la limitación a las propiedades latifundistas en al menos un 35\% de su extensión serviría a los fines del desarrollo de planes colonizadores.

Considerando que Misiones es un territorio fronterizo situado en las márgenes del país, la obra de Eidt nos induce a conocer más acerca de ese espacio, las particularidades de su poblamiento y las problemáticas vinculadas al mismo. En este sentido, es un libro que presenta puntos de contacto entre varias disciplinas: historia, geografía, economía, política y biología y representa una referencia prácticamente obligada para toda investigación que centre su interés ya sea en el poblamiento de Misiones como en la evolución del principal cultivo de esas tierras: la yerba mate. Al mismo tiempo, el empleo de un importante repositorio cartográfico que acompaña el relato histórico evidencia las dinámicas de construcción de poder regional, nacional e internacional que suscitaron las distintas etapas de la organización territorial de Misiones, en el extremo noreste de Argentina.

\section{Referencias bibliográficas}

Bartolomé, L. (1982). Colonias y Colonizadores en Misiones. Posadas, Instituto de Investigación Facultad de Humanidades y Ciencias Sociales, UNaM.

Bartolomé, L. (2000). Los Colonos de Apóstoles. Posadas, Editorial Universitaria.

Bolsi, A. (1986). "Misiones (una aproximación geográfica al problema de la yerba mate y sus efectos en la ocupación del espacio y el poblamiento)". Folia Histórica del Nordeste, Resistencia, IIGHI-CONICET-FUNDANORD, (7), 9-253.

Eidt, R. (1965). "Die Staatlishe und Private Besiedlung von Misiones (Argentinien)". Geographische Rundschau, 17, 464-470.

Gallero, M. C. (2009). Con la patria a cuestas. La inmigración alemana-brasileña en la colonia Puerto Rico-Misiones. Buenos Aires: Araucaria Editora.

Perie de Schiavoni, A. y Zouvi, S. (1985). La Colonización en Misiones: $1^{\circ}$ Parte (Contexto internacional, nacional y sus antecedentes). Posadas, Centro de investigaciones histórico-Culturales, Instituto de Investigación, Facultad de Humanidades y ciencias sociales, UNaM.

Zang, L. M. (2020). "La yerba mate como cultivo poblador: desde la decadencia de los yerbales nativos al auge de los yerbales implantados". Apuntes revista de Ciencias Sociales 47(87). https://doi.org/https://doi.org/10.21678/apuntes.87.975. 